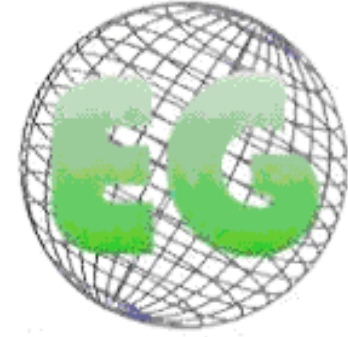

ISSN 1695-6141 N028

www.um.es/egloball

\title{
CLÍNICA
}

\section{Aplicación del proceso de enfermería en la atención domiciliaria de personas con enfermedad mental}

Application of home care nursing for people with mental illness

\section{${ }^{*}$ Castaño Mora, Y., "*E Erazo Chávez, IC., ***Piedrahita Sandoval, LE.}

\begin{abstract}
*Enfermera Máster en Salud Mental con énfasis en Ciencias Humanas y Sociales. Coordinadora Programa de Atención Domiciliaria Psiquiátrica. Hospital Psiquiátrico Universitario del Valle. Profesor Asistente. Área de Salud Mental y Psiquiatría. Programa de Enfermería. Facultad de Ciencias de la Salud. Universidad Libre Cali. E-mail: yamicamo@hotmail.com **Estudiante Escuela de Enfermería. Facultad de Salud. Universidad del Valle. ***Profesor Asociado. Área de Salud Mental y Psiquiatría Escuela de Enfermería. Facultad de Salud. Universidad del Valle.
\end{abstract}

Palabras clave: cuidado de enfermería; Atención Domiciliaria de Salud; salud mental; esquizofrenia.. Keywords: care; home health care; mental health; schizophrenia.

\section{RESUMEN}

La aplicación del proceso de atención de enfermería es un garante de calidad en los procesos asistenciales; no obstante, su uso en el cuidado de personas con enfermedad mental es muy limitado.

Objetivo: Mejorar la calidad en el cuidado de personas con enfermedad mental, mediante la aplicación de conocimientos teóricos y el desarrollo de destrezas, desde la implementación del Proceso de Atención de Enfermería en un Programa de Atención Domiciliaria de una institución de salud mental de tercer nivel del Suroccidente Colombiano.

Método: Investigación descriptiva transversal; participaron 38 personas con diagnóstico de esquizofrenia. Se utilizó la valoración según los patrones funcionales de salud de Marjory Gordon, en el período de enero - junio de 2010.

Resultados: Se identificaron 4 patrones alterados con prevalencia por encima del 20\%: Actividad y ejercicio, nutricional metabólico, rol relaciones y cognoscitivo sensorial. La intervención de enfermería estuvo orientada desde enfoques promocionales y preventivos, con cuidados específicos según requerimiento del paciente.

Discusión: Se requiere incentivar la aplicación del Proceso de Atención de Enfermería en la población de personas con enfermedad mental para garantizar un cuidado integral y de calidad, que busque involucrar la familia como elemento dinamizador del proceso de recuperación del sujeto intervenido. 


\section{ABSTRACT}

The application of nursing is a guarantee of quality healthcare processes; however its use in the care of people with mental illness is very limited.

Objective: To improve the quality of the care for people with mental illness through the application of theoretical knowledge and the development of skills gained from the implementation of nursing process in a home care program of a third level mental health Institution in the Colombian Southwest.

Method: descriptive transversal research; 38 people diagnosed with schizophrenia participated. The evaluation according to the functional patterns of health by Marjory Gordon was used, from the period of January to June 2010.

Results: 4 altered patterns maintained above 20\% were identified: activity and exercise, metabolic nutrition, relation and cognitive sensory roles. The intervention of nursing was oriented from promotional and preventive approaches with specific care according to requirements of the patient.

Discussion: It is required to encourage the application of nursing process in the population of people with mental illness to ensure a whole and quality care, which seeks to involve the family as a dinamic element of recovery process of the subject involved.

\section{INTRODUCCIÓN}

La salud mental es definida como el estado de bienestar por medio del cual los individuos reconocen sus habilidades, son capaces de hacer frente al estrés normal de la vida, trabajar de forma productiva y contribuir a la comunidad ${ }^{(1)}$. Las condiciones del entorno y las interacciones de los individuos pertenecientes a un determinado grupo social pueden generar alteraciones que dan lugar a la aparición de trastornos mentales. A nivel mundial los trastornos mentales alcanzan $14 \%$ de la carga global de enfermedad; por ser dichos trastornos algo tan debilitante, influyen más en dicha carga mundial que las enfermedades cardíacas, apoplejía o cáncer ${ }^{(2)}$ : Se prevé que para el año 2020, la depresión ocupará el segundo lugar dentro de las 10 principales causas de discapacidad en el mundo ${ }^{(2)}$.

En las Américas, 150 millones de personas tienen trastornos mentales o problemas psicosociales ${ }^{(3)}$. En Colombia, el Estudio Nacional de Salud Mental del año 2003 muestra que la prevalencia de cualquier trastorno mental es de $40 \%$, siendo las alteraciones más frecuentes: la ansiedad con 19,3\%, los trastornos afectivos $15 \%$ y el trastorno por uso de sustancias $10.6 \%{ }^{(4)}$. Dos de cada cinco personas presentan al menos un trastorno mental en algún momento de su vida, evidenciándose que la Región del Pacífico Colombiano tiene una prevalencia más elevada de trastornos afectivos en comparación con el resto del País. Adicionalmente, el estudio menciona que sólo 1 de cada 10 personas con trastornos mentales recibieron atención en el momento en que lo requirieron.

En Cali, para el año 2008 el Trastorno de Ansiedad tuvo una prevalencia del 34\% según el total de la consulta realizada, seguido de los trastornos del estado del ánimo con un $20 \%$ y consultas asociadas a trastornos pedagógicos en la población infantil y adolescente con el $11 \%{ }^{(4)}$. En el año 2010, el municipio reportó un número de suicidios de 94, lo que evidenció un incremento del $16 \%$ en el índice de autoagresiones en comparación con el año $2009^{{ }^{(5)}}$.

En la actualidad, los procesos de intervención dirigidos a la persona con enfermedad mental y su grupo familiar, se fundamentan en la articulación del trabajo interdisciplinario. Es de resaltar el papel que desempeña el profesional de Enfermería por la posición privilegiada que asume en el proceso de cuidado, por cuanto tiene la posibilidad de tener contacto permanente con las personas implicadas en el mismo. 
De ahí la importancia que el aporte del profesional de Enfermería sea congruente no sólo con la condición de salud de la persona sino que sus cuidados se fundamenten en un proceso científico que garantice la calidad de los mismos.

En su práctica asistencial, el profesional de Enfermería utiliza el Proceso de Atención de Enfermería (PAE), definido como un conjunto de acciones intencionadas que se realizan en un orden específico con el propósito de asegurar que la persona reciba los mejores cuidados por parte de los profesionales de Enfermería. Es un método sistemático y organizado, para realizar cuidados individualizados de acuerdo con el enfoque de cada persona o grupo de personas que responden a una alteración potencial o real de su salud ${ }^{(6)}{ }^{(7)}$. La inclusión del PAE como estrategia de cuidado ha sido escasamente empleada por los profesionales de Enfermería en las diversas áreas de asistencia ${ }^{(8)}$ y particularmente en el área de salud mental ${ }^{(9) .}$

El compromiso social de las instituciones de salud mental en la actualidad ha posibilitado la implementación de modelos asistenciales de corte comunitario; es así como en el Hospital Psiquiátrico Universitario del Valle se desarrolla el Programa de Atención Domiciliaria, el cual se define como un servicio complementario dirigido a pacientes de estratos 1 y 2 de la ciudad de Cali, que no cuentan con ningún tipo de seguridad social y que poseen una red de apoyo familiar. Este programa estructura su intervención sobre varios componentes: psiquiátrico, familiar, educativo y ocupacional que favorecen la orientación de la persona con enfermedad mental y su familia de forma integral, el cual es desarrollado por personal Profesional de Enfermería ${ }^{(10)}$.

La inclusión del PAE en el Programa de Atención Domiciliaria está encaminada a estructurar y estandarizar el quehacer del profesional de enfermería para fortalecer la calidad del cuidado dirigido a las personas incluidas en el programa. EI PAE permite el abordaje de todos los elementos apropiados para garantizar el cubrimiento de las necesidades de la persona y su familia, teniendo en cuenta sus requerimientos reales y el empleo de estrategias de valoración, diagnóstico e intervención acordes a las tendencias actuales de Enfermería basadas en la evidencia científica del ejercicio profesional.

Dada la carencia que se tiene por parte de las instituciones de salud en general y de manera específica de las instituciones de salud mental, en relación con la aplicación del PAE y teniendo en cuenta, la influencia que ejerce el trastorno mental en los perfiles de morbi-mortalidad de la población, así como la importancia que cobra el rol del Profesional de Enfermería al interior del equipo interdisciplinario, el presente trabajo buscó implementar el PAE en el cuidado dirigido a las personas con esquizofrenia vinculadas al Programa de Atención Domiciliaria de una institución de salud mental de tercer nivel de atención del Suroccidente Colombiano.

\section{MATERIAL Y MÉTODO}

Estudio de tipo descriptivo de corte transversal ${ }^{(11)}$. De una población total de 140 pacientes, se tomaron 38 hombres y mujeres con diagnóstico de esquizofrenia adscritos al Programa de Atención Domiciliaria de una institución de salud mental de tercer nivel de atención del Suroccidente Colombiano pertenecientes a los estratos 1 y 2 , que asistieron de manera ininterrumpida al programa por un período mayor a seis meses, como criterios de inclusión. La baja adherencia al tratamiento farmacológico y psicoterapéutico y los múltiples reingresos hospitalarios son condiciones tenidas en cuenta para la vinculación de los pacientes al programa domiciliario. 
Se utilizó la Guía de Valoración por Patrones Funcionales en la persona con enfermedad mental la cual fue diseñada en el marco de la asignatura Salud Mental y Psiquiatría de la Escuela de Enfermería de la Universidad del Valle. A través de ésta guía en fase de desarrollo se identifica el estado salud-enfermedad de la persona y su familia, teniendo en cuenta datos de identificación general e información objetiva y subjetiva proveniente de los once patrones funcionales de Marjory Gordon ${ }^{(7)}$. El estudio se realizó en el período enero - junio de 2010.

La valoración de las condiciones y necesidades de las personas mediante la aplicación de la guía fue la primera etapa del estudio efectuada por las investigadoras, en las instalaciones de la institución de salud mental, previa obtención del consentimiento informado diligenciado por las personas con enfermedad mental y sus cuidadores de forma escrita. En la segunda etapa, las investigadoras hicieron el análisis de la información recolectada acerca de los patrones y elaboraron los diagnósticos de enfermería correspondientes. En la tercera etapa o de intervención se desarrollaron actividades educativas con enfoque de promoción y de prevención y se realizaron los cuidados de enfermería, a partir de los diagnósticos de enfermería planteados, de acuerdo a priorización de los patrones alterados.

Las actividades educativas con los pacientes, se realizaron en el transcurso de tres meses; por medio de 4 encuentros, con una duración aproximada de 75 minutos cada uno, coincidiendo con la fecha de entrega de medicamento mensual establecida por el programa. En cada sesión se abordaron los temas como un proceso de construcción conjunta de conocimientos a partir de situaciones identificadas a través de dinámicas como los grupos focales.

El estudio tuvo el aval del Comité de Ética de la Facultad de Salud de la Universidad del Valle; de acuerdo a las normas éticas vigentes en Colombia ${ }^{(12), 13)}$.

Los datos fueron analizados con estadística descriptiva; para su procesamiento y análisis se empleó el programa SPSS $1.0^{(14)}$.

\section{RESULTADOS}

Las características socio demográficas de las personas participantes en el estudio se muestran en el cuadro I; la edad estuvo entre 18 y 57 años, con un promedio de 34 años (IC 95\% entre 30.16 y 37.84), mediana de 28 y desviación estándar de 12.1. El $60.5 \%$ eran de sexo masculino, $50 \%$ pertenecían a la etnia mestiza, $89.5 \%$ procedían del municipio de Santiago de Cali. $6.8 \%$ eran solteros; $50 \%$ tenían nivel educativo de secundaria incompleta. $100 \%$ de las mujeres se desempeñaban en oficios domésticos, $81.6 \%$ de los hombres se dedican a actividades de economía informal como vendedor ambulante, jardinero, ayudante de construcción, ayudante de joyería y $7.9 \%$ se encontraban desempleados.

El rol de cuidador(a) de la persona lo ejercía la madre en $52.6 \%$ de ellos(as), la hermana en el $10.5 \%$, el padre en el $10.5 \%$ y otros familiares en menor proporción. Es importante destacar que $\mathbf{7 . 9 \%}$ de las personas refirió ejercer el (ella) mismo (a) este rol. 
Cuadro I. Características sociodemográficas de las personas participantes en el estudio

\begin{tabular}{|lll|}
\hline & Frecuencia & $\%$ \\
Edad (años) & & \\
$18-30$ & 19 & $50 \%$ \\
$31-50$ & 12 & $31.6 \%$ \\
$51-57$ & 7 & $18.4 \%$ \\
Sexo & & \\
Femenino & 15 & $39.5 \%$ \\
Masculino & 23 & $60.5 \%$ \\
Etnia & & \\
Mestiza & 19 & $50 \%$ \\
Afrodescendiente & 12 & $31.6 \%$ \\
Indígena & 7 & $18.4 \%$ \\
Estado civil & & \\
Soltero(a) & 33 & $86.8 \% \%$ \\
Casado(a) & 2 & $5.3 \%$ \\
Separado(a) & 2 & $5.3 \%$ \\
Viudo(a) & 1 & $2.6 \%$ \\
Nivel educativo & & \\
Primaria incompleta & 8 & $21.1 \%$ \\
Primaria completa & 4 & $10.5 \%$ \\
Secundaria incompleta & 19 & $50 \%$ \\
Secundaria completa & 7 & $18.4 \%$ \\
\hline
\end{tabular}

\section{Patrones alterados}

De los 11 patrones evaluados, se identificaron 8 alterados, 4 con prevalencia por encima del $20 \%$ como se aprecia en el cuadro II. Los patrones más prevalentes fueron: patrón de actividad y ejercicio que estuvo alterado en 16 personas $(42.1 \%)$, nutricional metabólico en 13 personas (34. 2\%), rol relaciones en 11 personas (28.9\%) y cognoscitivo sensorial en 8 personas (21\%). No se identificó alteración en los patrones: sueño y descanso, tolerancia y enfrentamiento al estrés y valores y creencias.

Cuadro II. Caracterización de los patrones funcionales de las personas participantes en el estudio $\mathrm{n}=\mathbf{3 8}$

\begin{tabular}{|c|c|c|}
\hline \multirow{2}{*}{\multicolumn{3}{|c|}{ Patrón }} \\
\hline & & \\
\hline $\begin{array}{l}\text { Percepción y manejo de la } \\
\text { Salud }\end{array}$ & 6 & $15.8 \%$ \\
\hline Nutricional metabólico & 13 & $34.2 \%$ \\
\hline $\begin{array}{l}\text { Eliminación urinaria e } \\
\text { intestinal }\end{array}$ & 3 & $7.9 \%$ \\
\hline
\end{tabular}




\begin{tabular}{|c|c|c|}
\hline Actividad y ejercicio & 16 & $42.1 \%$ \\
\hline Sueño y descanso & 0 & $0 \%$ \\
\hline Cognoscitivo sensorial & 8 & $21 \%$ \\
\hline $\begin{array}{l}\text { Autopercepción } \\
\text { autoconcepto }\end{array}$ & y 2 & $5.3 \%$ \\
\hline Rol relaciones & 11 & $28.9 \%$ \\
\hline Sexualidad y reproducción & 2 & $5.3 \%$ \\
\hline $\begin{array}{l}\text { Tolerancia } \\
\text { enfrentamiento al estrés }\end{array}$ & y 0 & $0 \%$ \\
\hline Valores y creencias & 0 & $0 \%$ \\
\hline
\end{tabular}

\section{Diagnósticos de Enfermería}

A partir de la identificación de los patrones alterados con prevalencia por encima del $20 \%$ se formularon los diagnósticos de enfermería según la taxonomía NANDA, ${ }^{(15)}$ así:

Patrón de actividad y ejercicio: Déficit de actividades recreativas relacionado con entorno desprovisto de actividades recreativas, evidenciado por carencia de realización de actividades deportivas y pasatiempos habituales.

Patrón nutricional metabólico: desequilibrio nutricional por exceso relacionado con aporte excesivo según las necesidades metabólicas, evidenciado por peso corporal superior al ideal de acuerdo al índice de masa corporal.

Patrón de rol-relaciones: 1) Deterioro de la comunicación verbal relacionado con barreras psicológicas (psicosis, falta de estímulos), evidenciado por dificultad para mantener el patrón de comunicación habitual ,2) Deterioro de la interacción social relacionado con alteración en los procesos del pensamiento y trastorno del auto concepto evidenciado por interacción disfuncional con personas del entorno social en general.

Patrón cognoscitivo sensorial: Trastorno de los procesos del pensamiento relacionado con enfermedad mental, evidenciado por pensamiento inadecuado no basado en la realidad.

A partir de los diagnósticos de enfermería planteados se implementaron las siguientes intervenciones dirigidas a la persona con enfermedad mental: 1) asesoramiento nutricional a través de la actividad educativa "alimentación saludable en la persona que recibe psicofármacos", 2) terapia de actividad mediante el taller "ocupación del tiempo libre en casa", 3) apoyo en toma de decisiones y escucha activa a partir del conversatorio "comunicación asertiva" y 4) Manejo de la conducta, con la actividad "conociendo la enfermedad mental". 
La evaluación de las intervenciones se realizó al finalizar cada actividad, por medio de la aplicación de un formato elaborado por las investigadoras, el cual estimaba 4 aspectos: el nivel de disposición para la participación en actividades lúdico-reflexivas, la magnitud de la participación en la actividad y la apropiación de los conceptos; también, se generó un espacio abierto para el planteamiento de reflexiones adicionales. Se encontró que el $55 \%$ de las personas refirieron disposición para vincularse en las actividades lúdico-reflexivas, 64\% evidenciaron en gran magnitud su participación en las diferentes actividades y 45\% demostraron un nivel de apropiación fuerte de los conceptos abordados. Las personas expresaron algunas reflexiones adicionales respecto a su participación así: "es muy importante la participación en estas actividades", "se fortalece el programa de atención domiciliaria" y "me siento muy a gusto participando".

\section{DISCUSIÓN}

El presente estudio estuvo conformado predominantemente por personas jóvenes (18- 30 años) con diagnóstico de Esquizofrenia, lo cual coincide con lo mencionado por diferentes autores quienes señalan que la enfermedad mental afecta poblaciones jóvenes generando impacto sobre la capacidad de producción integral del individuo. De acuerdo con la literatura, el diagnóstico de esquizofrenia para el hombre se presenta entre los 15-25 años, con un pico hacia la mitad de la veintena mientras que en la mujer es entre 15- 30 años, con un pico algo más tardío y un pico adicional entre los 45 y 49 años (16). La predominancia del género masculino entre las personas del estudio también es consistente con el perfil epidemiológico reportado en la literatura a nivel nacional y mundial para trastornos psicóticos, específicamente esquizofrenia, ya que los estudios indican una mayor incidencia en los hombres ${ }^{(16)}$.

El estado civil de las personas evidenció una alta prevalencia de la condición de ser soltero, hecho que se asocia a la disfuncionalidad social derivada de la enfermedad, lo que condiciona un nivel de dificultad para la generación de relaciones de pareja estables en un individuo diagnosticado con esquizofrenia. Lo anterior se presenta con mayor frecuencia en la población masculina, la que conformó predominantemente la muestra del presente estudio. Adicionalmente, la bibliografía reporta que las mujeres con esquizofrenia pueden estar casadas en mayor proporción que los hombres y tienen dos veces más probabilidades de tener descendencia ${ }^{(17)}$. Llama la atención que la disfuncionalidad social es una condición evidente en este tipo de personas, a tal punto que es considerada como una categoría dentro de los aspectos valorados por el Manual de Clasificación y Diagnóstico de las Enfermedades Mentales (DSM IVTR) de la Asociación Americana de Psiquiatría ${ }^{(18)}$.

El proceso educativo formal de las personas del estudio alcanzó la etapa denominada de educación básica media en forma incompleta, lo cual está acorde con la literatura que dice que los hombres tienen un ajuste premórbido menos eficiente y menos logros académicos ${ }^{(19)}$. La ocupación corresponde en un alto porcentaje a empleos que no proporcionan una estabilidad económica significativa, debido a la condición de vinculación temporal, situación que repercute en la calidad de vida de estas personas. En la población femenina predominó el desempeño de actividades domésticas; lo cual coincide con lo reportado en la literatura. Al respecto, Contreras García afirma que las personas diagnosticadas con un trastorno mental severo, sufren además de las discapacidades y dificultades de integración, las consecuencias del prejuicio social que existe hacia las enfermedades mentales y quienes las padecen. El principal 
ámbito de discriminación para la persona con enfermedad mental es el laboral con sus consiguientes repercusiones económicas ${ }^{(19)}$.

El estudio señala a la madre como la persona responsable del proceso de cuidado de la persona con enfermedad mental. Afanador y colaboradores identifican que los cuidadores familiares son en su mayoría mujeres y conforman la parte más importante de las redes de apoyo y soporte social de quienes están enfermos o incapacitados y desarrollan su potencial de cuidado de múltiples formas, lo cual concuerda con lo evidenciado a través del estudio ${ }^{(20)}$, señala adicionalmente que la mujer pese a los múltiples roles que desempeña y al poco apoyo con que cuenta, aunados a la escolaridad que posee, continúa asumiendo el cuidado de sus seres queridos ${ }^{(20)}$.

Con relación a los patrones funcionales, se encontró que el patrón de actividad y ejercicio muestra un alto nivel de alteración, lo cual puede tener relación con el efecto de la medicación psicofarmacológica sobre la funcionalidad motora del individuo, adicionalmente la presentación de sintomatología negativa, tiene alta relación con el estilo de vida de la persona y hace que la persona con diagnóstico de esquizofrenia muestre, de manera crónica, una menor disposición para la participación en actividades de tipo lúdico y recreativo. García de Eulate menciona que el mayor riesgo que las personas con esquizofrenia tienen de padecer co-morbilidad orgánica metabólica y/o cardiovascular tiene que ver con diferentes factores: la enfermedad misma, la toma de fármacos anti psicóticos y los estilos de vida poco saludables como una dieta desordenada, sedentarismo y una muy elevada tasa de tabaquismo. Además, refiere que la unión de estos malos hábitos con los efectos adversos del tratamiento con anti psicóticos provoca un gran aumento del riesgo de padecer enfermedades cardiovasculares y endocrinas ${ }^{(21)}$. Esta situación se agrava con la alteración del patrón nutricional metabólico, el segundo patrón más afectado en las personas del estudio, quienes presentaron conductas de exceso en la ingesta calórica o disminución significativa frente al consumo de alimentos según el medicamento. Rodríguez Artalejo y colaboradores, afirman que la administración de fármacos anti psicóticos en la esquizofrenia se ha asociado a un mayor riesgo de obesidad, diabetes mellitus tipo 2 y dislipemia y potencia el elevado riesgo cardiovascular que basalmente presentan estos pacientes ${ }^{(22)}$.

El patrón de rol relaciones se ve tradicionalmente afectado en una persona con enfermedad mental debido a las confrontaciones que puede tener con las personas que le rodean como producto de alteraciones afectivas e ideativas. De igual manera, la sintomatología negativa que prevalece en el curso del trastorno conlleva a eventos de aislamiento y disminución del contacto social significativo, por lo cual la persona puede perder habilidad de interacción social o eliminar por completo cualquier tipo de actividad de socialización. Jiménez y colaboradores señalan que el funcionamiento social es un concepto que se correlaciona a su vez con el pronóstico del nivel de recuperación del sujeto con trastorno mental, la calidad de vida del mismo e incluso los costos económicos derivados de su manejo a largo plazo. Evidentemente, pacientes con mejor funcionamiento social tendrán menor perfil de vulnerabilidad ante la enfermedad misma y su curso variado ${ }^{(23)}$.

El patrón cognoscitivo sensorial reportó un alto nivel de alteración posiblemente debido a que involucra la valoración de la esfera mental, la cual abarca las diferentes áreas sensibles de presentar afecciones en la persona con esquizofrenia. Aunque existe una alteración significativa de dicho patrón es importante reconocer el impacto sobre la disminución sintomática que se presenta en la población vinculada a 
estrategias de acompañamiento ambulatorio, no obstante, existe un porcentaje que puede continuar presentando de manera crónica síntomas de la enfermedad, tal como lo reporta la bibliografía, así como también, experimentar fluctuaciones en niveles de recuperación, pasando de un estadio de resolución sintomática a un estadio de crisis.

La implementación del proceso de atención de enfermería permitió una vez identificados los patrones alterados y la elaboración de los diagnósticos prioritarios, establecer una serie de actividades de intervención desde la educación en salud. La realización de estas actividades evidenció un alto nivel de participación de las personas y la apropiación de los conceptos abordados, así como también nivel de gratificación desde la vinculación con la actividad y el aporte positivo que estas estrategias implican para el programa domiciliario institucional. El empleo de estrategias educativas puede verse limitado en población con diagnóstico de enfermedad mental, sin embargo, este tipo de intervención enfermera, permite no sólo favorecer el proceso de recuperación integral de la persona con enfermedad mental, sino también innovar frente al uso de procesos de acercamiento.

\section{CONCLUSIONES}

De acuerdo con los resultados obtenidos y lo evidenciado en la revisión de literatura, se encontró que la esquizofrenia es una enfermedad mental que afecta principalmente a población masculina en edades tempranas del ciclo vital, generando consecuencias negativas sobre los procesos de socialización del individuo que terminan por ocasionar disfuncionalidad en el establecimiento de dinámicas de interacción afectiva y social, así como también, interrupción de los procesos académicos y escasa vinculación con procesos ocupacionales y/o laborales productivos, que van en detrimento de las condiciones económicas de este grupo poblacional, aunado al requerimiento de contar con la figura de un cuidador familiar sobre el que recae la responsabilidad frente a la recuperación de la persona con enfermedad mental.

Los resultados de la valoración de los patrones funcionales, evidencian que la enfermedad mental afecta no sólo la esfera mental y el perfil conductual de la persona; trascendiendo los límites de lo personal, hacia lo familiar y lo social. La identificación de los patrones funcionales y los diagnósticos de enfermería que se ven afectados con mayor frecuencia en la persona con enfermedad mental, permite al enfermero(a) propiciar intervenciones en pro del mejoramiento de las condiciones de vida del individuo; constituyéndose así el proceso de atención de enfermería como una estrategia fundamental del cuidado.

Esta investigación tiene un impacto significativo, ya que la población de personas con enfermedad mental y su familia, requieren de procesos de cuidado integral que trasciendan el trastorno mental e incluyan la valoración de otros elementos fundamentales para lo cual la valoración por patrones se constituye en un referente apropiado a la necesidad de cuidado integral.

El proceso de cuidado de enfermería, requiere el uso de estrategias de intervención educativa, que para las personas con enfermedad mental representa un aporte significativo frente a la apropiación de su proceso de recuperación, por lo cual, se debe favorecer el uso de este tipo de abordajes.

Es importante en el presente estudio tener en cuenta las limitaciones derivadas de la aplicación del instrumento de valoración por patrones funcionales que se encuentra 
en fase de desarrollo. La extensión del mismo ocasionó alteraciones en la atención de algunos de los participantes, adicionalmente la condición de enfermedad mental, influye en la tolerancia sobre los tiempos de ejecución de actividades. Por lo anterior, se requiere la validación del instrumento de recolección de información teniendo en cuenta las características propias del grupo poblacional.

Son muy pocas las investigaciones que sobre la implementación del proceso de atención de enfermería, se realizan en población con trastorno mental, por lo que no permite una comparación adecuada de los resultados en relación con otras investigaciones. De allí la importancia de realizar un llamado al personal de enfermería, para fomentar los procesos investigativos en el área relacionados con la aplicación del proceso de enfermería.

Se sugiere continuar con estudios desde enfermería que favorezcan la integración de los conceptos teóricos del PAE en entornos prácticos comunitarios y clínicos, particularmente en el área de Salud Mental y Psiquiatría, fortaleciendo así la identificación del quehacer del profesional de enfermería y por consiguiente del componente disciplinar de la profesión.

\section{Agradecimientos}

Las autoras agradecen a los pacientes y familias, vinculadas al Programa de Atención Domiciliaria, así mismo a las directivas del Hospital Psiquiátrico Universitario del Valle, por su participación y apoyo para el desarrollo de la investigación.

\section{REFERENCIAS BIBLIOGRAFICAS}

1. Organización Mundial de la Salud. Informe sobre la salud en el mundo. Francia: OMS; 2001.

2. Prince, M. Patel, V. No health withouth mental health. (Fecha de acceso junio 23 de 2011) Disponible en: http://www.thelancet.com/journals/lancet/article/PIISO140673607612380/fulltext

3. Organización Panamericana de la Salud. La protección de la salud mental y la promoción de formas de vida sanas. Washington. OPS; 2002.

4. Ministerio de la Protección Social República de Colombia. Estudio Nacional de Salud Mental. Bogotá: Ministerio de la Protección Social; 2003.

5. Secretaria de Salud Pública de Cali. Boletín Informativo Volumen 12. Enero Diciembre de 2010. Disponibel en: www.cali.gov.co

6. Iyer, P. Taptich, D. Bernocchi, L. Proceso y Diagnostico de Enfermería. Madrid. McGraw-Hill Interamericana; 1997.

7. Escobar, G. Salud Mental Proceso de Atención de Enfermería. Manizales: ARS Ediciones; 2001.

8. Rojas, JG. Pastor P. Aplicación del Proceso de Atención de Enfermería en cuidados intensivos. Invest. Educ. Enferm. 2010; 28 (3): 223 - 35.

9. Echeveste, A. Planes y cuidados de enfermería en un centro de Salud Mental Comunitario. (Fecha de acceso junio 23 de 2011). Disponible en: http://www.anesm.net/contents/html.php?archivo= fcontinuada planesycuidadoscsm.

10. Castaño, Y. Guía de Atención Domiciliaria Psiquiátrica. (Fecha de acceso junio 1 de 2011) Disponible en: http://192.168.0.10/calidad/Procedimientos/PDF\%20Intervención\%20Social\%20y\%20Comunit aria/Atenciòn\%20Domiciliaria\%20Psiquiatrica\%20ISC.pdf. 2010. 
11. Polit, D. Hungler, B. Investigación científica en ciencias de la Salud. Sexta edición. México: McGraw-Hill; 2000.

12. Ministerio de Salud. Resolución 8430 de 1993. Bogotá, DC: Ministerio de Salud de la República de Colombia; 1993.

13. Ministerio de la Protección Social. Ley 911 de 2004. . Santa fe de Bogotá, DC: Ministerio de la Protección Social; 2004.

14. SPSS for windows 13.0 Chicago SPSS Inc., 2005.

15. NANDA -I. Diagnósticos Enfermeros: Definiciones y clasificación 2007 - 2008. España: Elsevier; 2008.

16. Vásquez, A. Jiménez, R. Esquizofrenia y género. Servicio Andaluz de Salud Apuntes de Psicología. 2006; 24 (1 - 3):157- 83.

17. Gaviria, S. Alarcón, R. Psicopatología y género: visión longitudinal e histórica a través del DSM. Rev. Colomb. Psiquiat. 2010; 39 (2): 389.

18. American Psychiatric Association. Diagnostic and statical manual of mental disorders. 4a ed. Washington, DC: American Psichiatric Association; 2002.

19. Contreras, V. Trabajo y Enfermedad Mental. (Fecha de acceso abril 19 de 2011); Disponible

en: http://www.fundacionsalto.org/pdf/colaboraciones/TRABAJO\%20Y\%20ENFERMEDAD\%20ME NTAL.pdf.

20. Pinto, N. Barrera, L y Colaboradores. Reflexiones sobre el cuidado a partir del programa: cuidando a los cuidadores. Aquichan AÑO 2005; 1(5): 129.

21. Eulate, C. Durán, M y cols. Grupo psicoeducativo sobre hábitos de vida saludables para personas con esquizofrenia en un hospital de día psiquiátrico; (Fecha de acceso abril 19 de 2011). Disponible en: http://www.unedtudela.es/uned/publicaciones/humanidades/extraordinario08-articulo4.pdf.

22. Rodríguez, F. Bacab, E y Cols. Grupo de trabajo de la Sociedad Española de Diabetes, Sociedad Española de Endocrinología y Nutrición, Sociedad Española para el Estudio de la Obesidad y Sociedad Española de Psiquiatría. Med Clin (Barc). 2006; 127(14):542-8.

23. Jiménez, R. García, A. Habilidad social y funcionamiento social en pacientes con esquizofrenia. Apuntes de Psicología. 2004; 22 (1): 111-20. 